\title{
Cooperation in Games with Forgetfulness
}

\author{
Raphael Thomadsen $^{\mathrm{a}}$ and Pradeep Bhardwaj ${ }^{\mathrm{b}}$
}

November 2008

JEL code: C72

${ }^{a}$ Corresponding author. UCLA Anderson, 110 Westwood Plaza, Suite B411, Los Angeles, CA 90095. raphael.thomadsen@anderson.ucla.edu. T: (310) 794-2100. F: (310) 206-7422.

${ }^{\mathrm{b}}$ Kenan-Flagler Business School, University of North Carolina, McColl Building, Chapel Hill, NC 27599.

Pradeep_bhardwaj@unc.edu. T: (919) 962-4926. 


\title{
Cooperation in Games with Forgetfulness
}

\begin{abstract}
Companies and managers are apt to forget information, yet game theory assumes that all players have perfect recall. This paper expands the literature by examining how introducing forgetfulness into a multi-player game-theoretic framework can help or hinder cooperative behavior. We distinguish between forgetting histories and forgetting strategies, and explain how classic game theory models and equilibrium concepts should be adapted to accommodate imperfect recall. We find that forgetfulness impacts the ability of firms to cooperate in countervailing directions. On the one hand, forgetfulness can diminish the ability to punish deviators, making cooperation more difficult. On the other hand, forgetfulness can make meting out severe punishments credible, and if the players forget their strategies then forgetfulness can also decrease the ability for players to effectively deviate, facilitating cooperation. When players forget their strategies, their reduced ability to deviate may be so severe that the equilibrium payoff may be below the minimax
\end{abstract}




\section{Introduction}

While the game theory literature classically assumes perfect recall, players in economic games often forget the past. This forgetfulness occurs partly due to the imperfect behavior of people, who forget past information. Given that managers in firms are humans, and thus subject to human fallibility, it then follows that understanding the role of forgetfulness is important for analyzing strategic interactions in business.

For firms, perfect recall is also synonymous with assuming that information is perfectly transferred across divisions and generations of leadership. One can therefore argue that firms are even more likely to forget than individuals due to the decentralization of firms' operations across time and divisions. The importance of this information loss is evidenced by the large literature on knowledge management focusing on how to reduce this information loss (e.g., Stein 1995, Alavi and Leidner 2001, and Argote, McEvily and Reagans 2003). These losses also have practical implications on firms' decisions (see, Benkard 2000, Besanko, Doraszelski, Kryukov and Satterthwaite 2007).

For expositional purposes, we illustrate the context we have in mind with an example of two colluding firms. Imagine that product managers at two competing companies, A and B, have colluded in the market place for several years. The managers sustain this collusion through a Nash reversion punishment scheme. One day the product manager at company A retires. The product manager at company B is trying to figure out whether to continue his collusive conduct. Perhaps the retiring manager at firm A has briefed her successor on the collusive conduct between the two firms. On the other hand, the retiring manager might not have briefed her successor - either because she did not want to admit to acting in an illegal manner, or because she may have hundreds of these types of details that she manages and she may not have the time or the ability to fully brief her successor. Thus, the manager of firm B has uncertainty about whether the arrangement between the two firms has been passed on to the next generation of managers. Also, the manager of firm B has to figure out how the new manager will behave if the retiring manager has not briefed her successor about the arrangement.

This paper considers what happens to the ability of firms to cooperate when

institutional forgetfulness occurs. We focus on two types of information loss. First, companies can lose knowledge of past historical events. We model this information loss 
as being complete in the model for simplicity, but in real life this likely corresponds to a lack of information transfer of key details. Second, managers can fail to pass along information about their strategy for a multitude of reasons. Perhaps the managers do not want to admit what their strategy was. For example, a manager might not want to discuss illegal collusive behavior. Alternatively, this information loss can happen because there are many different strategic decisions that each manager makes, and, because of people's limited ability to process information, the manager may not be able to pass along all of the details about their strategy for every project. Finally, decisions that are often modeled as a simple strategic decision (e.g., "collude") may require institutional support that can be lost in a transition from one management to the other. For example, when ADM set up a lysine price-fixing cartel, they had to figure out how to adhere to the collusive quotas without telling middle management or sales representatives what they were doing (Connor 2001). When there is a change in management, the details about how one implements such a collusive regime can be lost, effectively causing the firm to forget to collude.

A key question that arises in this analysis is what a firm does after it forgets its strategy. If we consider the case where the firm forgets its strategy due to a management transition, it seems reasonable to assume that the next manager will act in a way that managers would typically act in a certain situation. If managers are trained to approach competitors in an adversarial manner, the new manager would likely avoid collusion unless they had been briefed about the previous collusive relationship. In this paper, we call such behavior a "norm" and illustrate how the presence of these norms affects the ability of firms to cooperate.

We show that forgetfulness can either help or hinder cooperation. On one hand, forgetfulness makes it harder for the players to effectively punish deviators. On the other hand, forgetfulness can sometimes make severe punishments credible. Further, if the players forget their strategies then players who cheat on cooperation might forget their transgression and forget to defend against being punished. One result is that forgetfulness can also entrench inefficient marketing behavior. In fact, we find that forgetfulness could support on-the-path equilibrium payoffs that are below the minimax. 


\subsection{Key Issues and Prior Literature}

Despite the fact that most of the game theory literature assumes that players have perfect recall, the idea of having forgetful players is not new. Indeed, Von Neumann and Morgenstern (1953) discuss how to model forgetful players in their seminal book on game theory. They suggest that four players playing a game of bridge should be modeled as a two-player game, with each team being modeled as a single forgetful player, where the player imperfectly communicates information to themselves through signaling. ${ }^{3}$ They then discuss that the right way to model a forgetful player would be to split the player into multiple versions of themselves and restrict the information sets between them. ${ }^{4}$ While they acknowledge the issues that arise in this type of analysis, they never delve deeply into these issues.

This basic approach to modeling forgetfulness was used by Piccione and Rubinstein (1997). Their seminal work, and a series of papers discussing forgetfulness in game theory in an issue of Games and Economic Behavior, discusses issues that arise in considering strategic forgetfulness in single-player games (Aumann et. al. 1997, Battigalli 1997, Gilboa 1997, Grove and Halpern 1997, Halpern 1997, Lipman 1997, and Segal 2000). Much of the attention from these papers focuses on the case of the absentminded driver, where a single player forgets whether they have made a decision previously. (This is called "repeated decisions" by Alpern 1988.) We assume in our analysis that players have a calendar, so all players know which stage of the game they are playing. Nevertheless, most of the debate about how decisions with forgetfulness should be modeled and analyzed applies even in cases without repeated decisions.

One key issue raised in this literature is whether information about a player's strategy is conveyed between the different versions of themselves in the game, and whether the different incarnations of the player can coordinate their strategies. Piccione and Rubinstein consider the case where the players cannot communicate changes in their strategy to future selves and call such an approach multiself consistent. Battigalli, extends this concept by defining a modified multiselves sequential equilibrium, which states that players cannot change the strategies for their future selves. However, the

\footnotetext{
${ }^{3}$ See Sec. 6.4 .2 of their book.

${ }^{4}$ See Sec. 12.2.2 of their book.
} 
concept of modified multiselves consistency does not answer how the strategies between the different selves are set. Piccione and Rubinstein consider the case where the strategies are not coordinated between different incarnations of an individual. In contrast, Aumann et al. (1997), propose that strategies are set during a planning stage, but that the different incarnations cannot communicate beyond that point. Also, the plan set in that planning stage must be consistent with beliefs at the action stages, which are the decision nodes in the game.

Our approach mirrors this discussion, but extends the analysis to multi-player games. We consider two cases of forgetfulness. In one case, we assume that players have a pre-game planning stage where they set their strategy for all future versions of themselves. While the players can coordinate their strategy across the different versions of themselves at the beginning of the game, they cannot change the strategies of future selves later in the game; we will call this forgetting histories but not strategies. The equilibria that arise from these assumptions are in the spirit with the concept of forgetfulness found in Aumann et al., and Halpern.

In the other case we consider, players have no planning stage over which they can communicate a strategy to future versions of themselves. We call this forgetting strategies and histories. The equilibrium outcomes that come from these assumptions are in the spirit with the concept of forgetfulness found in Rubinstein and Piccione.

In order define our equilibrium concept, we start with the formalization of a game as defined in Mas-Colell et al. (1995, p. 227). In this formalization, a game is specified as $\Gamma=\{\chi, A, I, p(\cdot), \alpha(\cdot), h, H(\cdot), i(\cdot), \rho(\cdot), u\}$, where $\chi$ represent nodes, $A$ a set of possible actions, and $I$ represents the players in the game, $\{1, \ldots, I\}$. The terms $p$ and $\alpha$ are functions that define the structure of the game tree. $h$ is a collection of information sets, and $H: \chi \rightarrow h$ assigns each decision node $x$ to an information set $H(x) \in h . \quad$ i: $h \rightarrow\{1, \ldots$, $I$ ) assigns each information set to a player, $\rho$ represents nature's moves, and $u$ is a payoff function assigned to terminal nodes.

We modify the game as follows. First, we partition each of the nodes belonging to player $I$ as belonging to one of $K$ incarnations of themselves. Thus, the set of players could be modified as $\left\{1_{1}, \ldots, 1_{K 1}, \ldots, I_{1}, \ldots, I_{K I}\right\}$. Each information set is them assigned to one particular incarnation of one particular player. Thus, $i: h \rightarrow\left\{1_{1}, \ldots, 1_{K 1}, \ldots, I_{1}, \ldots\right.$, 
$\left.I_{K I}\right\}$. Also, we assume perfect recall within each incarnation of the same individual. That is, consider nodes $x, x^{\prime}, y, y^{\prime}$ belonging to incarnation $J_{k}$, where $x$ is a (possibly indirect) predecessor to $y$ and $x^{\prime}$ is a predecessor to $y^{\prime}$. Then $H(y)=H\left(y^{\prime}\right)$ only if $H(x)=$ $H\left(x^{\prime}\right)$ and the action at $x$ that leads (possibly indirectly) to node $y$ is the same as the action at $x$ ' that leads to node $y^{\prime}$.

Let $\beta$ denote the behavioral strategies of the players, where $\beta$ assigns a probability to action $a \in A(x)$ for any node $x^{\prime}$ such that $H\left(x^{\prime}\right)=H(x)$. Define $\mu$ as a belief, where $\mu(x \mid h)$ represents the probability that the player attributes to the possibility of their being at node $x$ conditional on being at information set $h$.

Following Battigalli, we say that an assessment $(\mu, \beta)$ is weakly consistent if for all information sets $h$ and $x \in \chi, \mu(x \mid h) p(h \mid \beta)=p(x \mid \beta)$, where $p$ denotes a probability. We then use Battigalli's definition of a modified multiselves sequential equilibrium to be a set of $(\mu, \beta)$ such that

(1) $\forall a, a^{\prime} \in A(h), \beta(a \mid h)>0 \Rightarrow \mathrm{E}(u \mid h, a ; \mu, \beta) \geq \mathrm{E}\left(u \mid h, a^{\prime} ; \mu, \beta\right)$

(2) There exists a sequence of strictly positive weakly consistent assessments $\left(\left(\mu_{k}\right.\right.$, $\left.\left.\beta_{k}\right)\right)_{\mathrm{k} \geq 1}$ such that $\lim _{\mathrm{k} \rightarrow \infty}\left(\mu_{k}, \beta_{k}\right)=(\mu, \beta)$.

We restrict our analysis in this paper to modified multiselves sequential equilibria. We assume that information that is learned by one incarnation of a player is not transferred to other incarnations of the player. Thus, a forgetful player always forgets the history of the game. In our framework, we only consider two-period games, with each period having a simultaneous-choice stage game. Thus, this restriction means that if a player is forgetful they will not be able to observe what actions were played in the first period of the game.

As noted above, we consider two possibilities about how the behavioral strategies are chosen within this framework. In one case, the strategies are chosen in a planning stage before the game begins, consistent with the conceptualization of forgetfulness advocated by Aumann et al. Once play begins, however, a player cannot change the strategies of future incarnations. This restriction is required so the player cannot signal the past history to themselves and undo any forgetfulness in the past history of the game. Of course, an equilibrium of this type could only have a profitable deviation across incarnations in response to information which is later forgotten, because any other 
profitable coordination could be attained during the planning phase. We call this way of choosing strategies "forgetting histories, not strategies."

The other way one could model the choice of strategies is that each incarnation of the players could select their strategies without communicating with their other incarnations. This assumption might be more reasonable than the assumption of a planning phase if the game with forgetfulness is used to model the actions of firms or organizations and where the forgetfulness reflects a transfer in management with an imperfect transfer of information. In such a case, each incarnation selects a complete strategy, which includes choices for information sets for other incarnations. This allows the player to analyze how, for example, future incarnations will respond to the player's current actions. In equilibrium, each of these incarnations will make the correct inference about how the different incarnations of themselves will behave. However, the player cannot coordinate deviations between the different incarnations of the players. This can change the way the game will play out. For example, a player might wish to cooperate with their opponent today conditional on being able to cooperate in the next period, but if the player knows that the next incarnation of themselves will be non-cooperative, they may instead choose not to cooperate. Also, conditional on the player not cooperating in period 1, being non-cooperative can be the optimal action for the player in period 2. In this paper, we will call this later type of forgetfulness "forgetting strategies and histories."

One issue that arises in this latter type of forgetfulness is how the players assess what the strategies of the other incarnations of themselves will be. In considering this, we consider a similar question of how players know the equilibrium strategies of the other players in games of perfect recall. In perfect recall, one interpretation of the equilibrium is that the equilibrium is the result of pre-play negotiation, where players announce their strategies before the game as cheap talk, but where these strategies form equilibria because they are self-enforcing (Binmore 1992, p. 305, Mas-Colell et. al. 1995, p. 249). This interpretation of how equilibria get selected is consistent with the formation of strategies with forgetfulness when we allow for a planning phase of the game. However, when we consider the case of players forgetting strategies and histories, we explicitly rule out communication of the strategies between the different incarnations of 
the players. In this case, we must consider the equilibrium strategies within incarnations as being set through one of the other mechanisms considered in the game theory literature. Mas-Colell et al. suggest focal points or stable social conventions as other ways equilibrium strategies may be coordinated. We follow this suggestion and give the term "norms" to these focal points or social conventions, which we believe is a relatively natural way to think about how managers at a firm will assume that future managers will behave. That is, the players assume that the other incarnations of themselves (the future and past managers of the firm) likely behaved in a way that managers would typically act in a certain situation. ${ }^{5}$

Before we summarize our results and lay out the outline of the paper, we also wish to acknowledge a literature that deals with forgetful consumers (e.g. Mullainathan 2002, Mehta, Rajiv and Srinivasan 2004, Chen, Iyer and Pazgal 2005, and Ofek, Yildiz and Haruvy 2007). However, in these papers there is no strategic interaction between the consumers. Consumers' forgetfulness can have an effect on the strategic interaction between the firms that supply the consumers; for example, in Chen et al., firms have a different pricing equilibrium than would emerge if consumers remembered prices perfectly. However, the strategic players themselves - the firms in Chen et al. - are not forgetful. ${ }^{6}$ Similarly, in Ofek, Yildiz and Haruvy (2007) a decision maker learns about their own valuation of a product, but then forgets some information about the signals that they received about their valuation, remembering only whether their valuation was above the market price at the time of the decision. However, the players do not adjust their current period actions to signal information strategically to their future selves. One important issue that this literature raises is whether the players are aware of the fact that they are forgetful. Consistent with Piccione and Rubinstein and many others, but in contrast with Mullainathan, we assume that players are aware of the fact that they may forget (and know when they have forgotten), and we allow players to account for this

\footnotetext{
${ }^{5}$ Though we do not push this point further, one could imagine that if all managers would approach a particular situation in the same manner then it would be reasonable to model the norm as a pure-strategy. On the other hand, if there is a lot of heterogeneity in how managers would approach a particular situation, one might want to consider mixed-strategy norms.

${ }^{6}$ Besanko et al. consider how forgetting knowledge of productive efficiency - as measured by marginal cost - affects how a market will evolve. However, firms in their model are not forgetful about anything related to their strategy.
} 
forgetfulness in their strategies.

Other papers related to ours include Bernheim and Thomadsen (2005), which also studies forgetfulness in a multi-player setting. However, in that paper forgetfulness has an impact only on players' strategy through their anticipatory emotions. Without these anticipatory emotions, the forgetfulness does not have an effect. In contrast, we assume that players are fully rational and do not have an emotional response to manage. ${ }^{7}$ Finally, Anderlini, Gerardi and Lagunoff (2007) consider a similar model of dynasties, where players live only one period but consider the payoffs of their prodigy when making decisions. The players never observe what happens in other periods, but they can send signals to the immediately succeeding generation. This model could be thought of as a model of forgetfulness - where forgetfulness occurs $100 \%$ of the time, although the goals, mechanics and results of that paper differ substantially from ours.

The outline of the remainder of the paper is as follows. Section 2 lays out the modeling framework used in most of the paper. In Section 3, we analyze cooperation with forgetfulness in histories and strategies. In Section 4, we analyze cooperation in games with forgetfulness in histories but perfect recall in strategies. Finally, Section 5 concludes.

\section{Model}

We analyze the effect that forgetfulness has on the ability of players to cooperate using a simple two-stage game framework. The players make simultaneous choices in the first stage, and the choices of the first stage are then revealed. Between the first and second stages, each player is forgetful with probability $\lambda \in(0,1)$. If the player is forgetful, they are replaced by a different incarnation of themselves; this incarnation has the same payoff as the individual, but there is no communication between the incarnations so no information from the first stage is passed to the next incarnation when the player forgets. The forgetfulness occurs independently among the players, so the probability that both players forget is $\lambda^{2}$. The players know whether they have been forgetful, but they cannot

\footnotetext{
${ }^{7}$ More broadly, our paper is also related to games with bounded rationality. For example, Rubinstein's (1986) paper about games with automata players could be construed as studying a different form of forgetfulness.
} 
observe whether their opponent has been forgetful. The players then play the second stage, which is assumed to have at least two Nash equilibria with different payoffs for the players.

Most of the analysis focuses on a fairly generic yet simple version of this game. The first stage of the game is assumed to have a cooperative outcome that gives better payouts for both players than any stage-Nash equilibrium gives, but which itself is not a stage Nash equilibrium. This first stage is not explicitly modeled in this paper because all that is important is the immediate gains in payoffs from cheating on the cooperative outcome - the other details about the first stage game are unimportant. The second-stage game consists of a symmetric $2 \times 2$ simultaneous move game pictured in Figure 1. Our goal is to use a simple, but fairly generic, game, with the key property being that this stage has two pure-strategy stage Nash Equilibria (due to the parameter restrictions), one with a high payoff and one with a lower payoff. In addition to the pure-strategy equilibria of $\mathrm{AA}$ and $\mathrm{BB}$, there is also a mixed-strategy Nash equilibrium where each player plays action A with probability $\frac{-b}{1-b-c}$. The effect of forgetfulness on the ability to cooperate can be analyzed through just the second stage of the game and examining the largest differences between average payoffs that are received after cooperation is sustained in the first stage compared to those sustained if cooperation is violated by either player in the first stage. ${ }^{8}$

\section{Forgetting both Strategies and Histories.}

We first consider cooperation in games where players forget both strategies and histories. We model players forgetting their strategies by assuming that players who forget their strategy follow a strategy that is dictated by a cultural or business norm. For example, if the norm is to act cooperatively, then when a player forgets the past he will play action A. In the case where forgetfulness is a result of human behavior, this assumption of following a norm can be justified by the idea that a forgetful player will behave in a way that this individual generally behaves when they face a similar scenario. In the case where forgetfulness arises due to a change in management, this intuition is

\footnotetext{
${ }^{8}$ Deviations by more than one player can be treated in any arbitrary self-enforcing manner.
} 
strengthened: the most reasonable belief about the future management's behavior is to assume that future management will behave the way managers typically behave when faced with similar situations. We allow the norm to be either a pure-strategy or a mixedstrategy norm. In the latter case, the randomness in the mixing can represent either true randomness or alternatively a distribution of types, where the old management does not know at the time of their first-stage decision the type of new management that will replace them if such a transition does occur.

We show that while cooperation is often easiest to sustain under cooperative norms, there exist conditions where cooperation can best be sustained under noncooperative norms. We then note that forgetfulness can either aid or obstruct the ability of the players to cooperate depending on the payoffs of the game. This tension is the result of the fact that while forgetfulness can hinder cooperation because players may forget to punish their opponents for deviations, forgetfulness can also facilitate cooperation because a player may avoid deviating for fear that they will forget to act defensively against punishment and because forgetfulness can make credible severe punishments which hurt both players. Finally, we demonstrate that these later effects can

dominate strongly enough that the on-the-path equilibrium payoffs can be below the minimax when there is some forgetfulness.

\subsection{A Simple Example}

To illustrate these effects, consider the two-stage game represented in Figure 2. The first stage of this game is a classic prisoner's dilemma, and the second stage of this game has three equilibria: (A, A) gives each player a payoff of 1, (B, B) gives a payoff of 0 , and a mixed-strategy equilibrium, where each player plays action A with probability $8 / 9$, and receives a payoff of $4 / 9$.

We first analyze whether cooperation can be sustained in the first period under the classic assumption of perfect recall. The equilibria of the second stage can be used to create a reward and punishment scheme for the first period. If both players play $\mathrm{A}$ if cooperation is sustained in the first period, and B if there were any deviations in the first period, then the best equilibrium $(\mathrm{A}, \mathrm{A})$ is given as a reward for cooperation and the worst equilibrium (B, B) is given as punishment. The difference in payoffs between this 
reward vs. punishment is 1 , which is less than $1.5(=5.5-4)$, the amount that players would gain from unilaterally cheating on the cooperative outcome in the first stage. Thus, the second-stage reward from cooperating in the first period is not large enough to prevent cheating, and there will be no cooperation in the first stage.

Now suppose that each player forgets their strategy and the history of the game between the two stages with a probability $\lambda$. We illustrate the example by setting $\lambda=1 / 2$. Then, the following strategies played by both players forms a modified multiself sequential equilibrium: In stage 1, play cooperate. In stage 2, the player plays $A$ if they are forgetful. If the player recalls the first stage, they play A if they recall cooperation, but play B if they recall any deviation.

We can verify this equilibrium by solving backwards. Suppose, first, that the player recalls cooperation being sustained in the first period. Then if the player plays A they can expect the other player to play A - regardless of whether the other player forgets the past or not - and get a payoff of 1 . On the other hand, if the player deviates to B then they will receive a payoff of only 1/2. Thus, playing A when the player recalls cooperation from period 1 is a best response. Note that if the player is forgetful between the two periods, they will assume that the other player and the previous incarnation of themselves played the equilibrium strategies. Thus, the calculations that support them playing $\mathrm{A}$ is the same as those for when the player recalls cooperation. ${ }^{9}$ Finally, suppose that the player recalls a deviation in the first period. If the player follows their strategy and plays $B$ then they will get a payoff of $1 / 2 \lambda$. On the other hand, if the player deviates and plays $A$, they will receive a payoff of $\lambda-4(1-\lambda)$, which is smaller than $1 / 2 \lambda$ as long as $\lambda<8 / 9$. Note that this last calculation demonstrates that a player will not "pretend to forget" the past in hopes of cooperating.

Given that the players will not deviate from their $2^{\text {nd }}$ period strategies, we analyze what the expected values of the $2^{\text {nd }}$ period payoffs are at the time of the first period

\footnotetext{
${ }^{9}$ Recall from Section 1.1 that the part of the strategy that occurs under recall and the part that occurs after forgetfulness are chosen by different incarnations of the players without any coordination. Thus, the recall part of the strategy must be optimal conditional on the forgetful incarnation's strategy, and the forgetful part of the strategy must be optimal conditional on the strategy of the incarnation with recall. We also consider how the results change when players have a planning stage where they set the strategies for all of their incarnations jointly, below.
} 
decision. If the players play cooperate, the players can then anticipate that (A, A) will be played in the $2^{\text {nd }}$ stage, and that they will receive a payoff of 1 . On the other hand, if the player unilaterally deviates in the $1^{\text {st }}$ stage, their expected $2^{\text {nd }}$ stage payoff is

$$
\lambda^{2}+\frac{1}{2} \lambda(1-\lambda)-4 \lambda(1-\lambda)
$$

where the first term represents the probability that both players forget the past, the second term reflects the payoff when only the other player forgets that there was a deviation, and the third term reflects the payoff when the deviator forgets that the game is in punishment mode. The last contingency, that both players recall the past and obtain zero, occurs with probability $(1-\lambda)^{2}$. When $\lambda=1 / 2$, the payoff in equation (1) becomes $-5 / 8$. Note that the difference in the $2^{\text {nd }}$ period payoffs in the cooperation state vs. the punishment state is now $1-(-5 / 8)=1.625$, which is enough to ensure cooperation in the first stage.

Thus, cooperation in stage 1 can be supported under forgetfulness, even while it cannot be supported under perfect recall. It may seem counter-intuitive to some readers that forgetfulness can aid cooperation given that a player who has been cheated on may forget to punish a deviator. However, in this example, this effect was more than offset by the risk that a deviator would take that they might themselves forget that they deviated in the first period and thus they may forget to defend themselves against a severe punishment. Later in this paper we will demonstrate that this last effect can be so strong that in some cases it may be possible to sustain below minimax payoffs.

We next consider what happens when players instead forget the past history of the game, but not their strategies. As noted in Section 1.1, this means that each player sets the strategy for all incarnations of themselves in an initial planning stage. In this case, cooperation is not achievable, as was true in the perfect recall case. The reason for the different result is that players are able to coordinate playing a deviation in the $1^{\text {st }}$ stage in the $2^{\text {nd }}$ stage action even when the player forgets by choosing the optimal strategies for all incarnations of themselves in a planning stage. To see that no equilibrium has $1^{\text {st }}$ stage cooperation, consider that the payoff of any such proposed equilibrium must be less than or equal to $5=4+1$ (the cooperation payoff, plus the greatest obtainable payoff for the $2^{\text {nd }}$ stage game). On the other hand, a player could profitably deviate from the proposed cooperation by unilaterally deviating to this strategy (which will be set in the 
planning stage): play Fink in the first period, play B in the second period regardless of what is remembered. ${ }^{10}$ The minimum payoff of this deviation would be 5.5 , since the minimum payoff that this player would receive in the second period, conditional on playing B, is zero. Thus, in this game it is impossible to support full cooperation when players forget the history of the game, but not their strategies. We chose this example to highlight the differences between the two different types of forgetfulness. However, we note later that forgetfulness in history but not strategies can sometimes aid cooperation.

\subsection{Impact of Imperfect Recall on the Ease of Cooperation}

We now return to considering the more-general two-stage game described in Section 2, where the second stage is represented by Figure 1. Note that $\mathrm{A}$ is the unique best response to an opponent playing action A with a probability greater than $\frac{-b}{1-b-c}$, while $\mathrm{B}$ is the unique best response to an opponent playing A with a lower probability. This implies that there are three Nash equilibria of the stage game: Both players could play A, both players could play B, or both players could mix, playing A with probability $\frac{-b}{1-b-c}$. The payoffs of these equilibria (to each player) are 1,0 and $\frac{-b c}{1-b-c}$, respectively.

Under perfect recall, the strongest equilibrium incentives for cooperation in the first stage result from strategies involving both players playing A when cooperation was sustained in the first period. The defection strategy that gives the strongest incentives is for both players to play B if $c>0$, or both players playing A with probability $\frac{-b}{1-b-c}$ if $c<0$. The firms will then cooperate in the first stage if the unilateral gains from deviating are less than $\operatorname{Max}\left(1,1+\frac{b c}{1-b-c}\right)$.

\footnotetext{
${ }^{10}$ This deviation need not be the most-profitable deviation; all that is required to prove that cooperation cannot occur in equilibrium is find a deviation that obtains higher profits than following the proposed equilibrium strategies. If there is a response to the opponent's strategy that provides a higher payoff than playing B provides, conditional on being in the state caused by the player's deviation, then the player should propose that response in the planning stage for both the recall and forgetful $2^{\text {nd }}$-stage contingencies.
} 
We examine how forgetfulness affects the ability of firms to cooperate by calculating how forgetfulness affects this maximum wedge between the expected payoffs that are earned when cooperation is sustained in the first stage compared to the expected payoffs that are earned when cooperation is violated by one player in the first stage.

The strategies for each player will be a 5-tuple: $\left\{P_{\mathrm{CC}}, P_{\mathrm{CD}}, P_{\mathrm{DC}}, P_{\mathrm{DC}}, P_{\mathrm{F}}\right\}$, where $P_{S}$ reflects the probability that the player will play action A if the state for that player is $S$. The five states they can face are $C C$ if the player recalls both players playing cooperate in period $1, C D$ if the player recalls player 1 cooperating but player 2 deviating in period 1 , $D C$ if the player recalls player 1 deviating but player 2 cooperating in period $1, D D$ if the player recalls both players deviating in period 1 , or $F$ if the player has forgotten what happened in period 1. We do not require the players to have symmetric strategies, so we denote the probability that player $i=1,2$ will play A in state $S$ as $P_{S i}$.

We begin by considering what probabilities can be supported on the equilibrium path for any equilibrium supporting cooperation in the first stage. Consider the strategy of player 1. Denote as $P_{1}$ :

$$
P_{1} \equiv(1-\lambda) P_{C C 1}+\lambda P_{F 1}
$$

$P_{1}$ represents the unconditional probability that player 1 will play action A in the second period if cooperation is sustained in the first period.

Consider three cases. First, if (2) is greater than $\frac{-b}{1-b-c}$ then the best response for player 2 is to play A with probability 1. Thus, $P_{C C 2}=P_{F 2}=1$. Note that the probability of playing $\mathrm{A}$ in the forgetfulness state must be a best response to the probability that the other player plays $\mathrm{A}$ on the equilibrium path. Given that player 2 plays A with probability 1 , it must also be that $P_{C C 1}=P_{F 1}=1$. Thus, one set of potential equilibrium strategies involves a fully cooperative norm and both players playing A with probability 1 on the path.

Second, suppose that $P_{1}$ is less than $\frac{-b}{1-b-c}$. Then the same logic dictates that $P_{C C 2}=P_{F 2}=0$, which in turn implies that $P_{C C 1}=P_{F 1}=0$. Thus, the second set of potential equilibrium strategies involves both players playing A with probability 0 on the equilibrium path. 
Finally, consider the case where $P_{1}=\frac{-b}{1-b-c}$. Then any set of actions is a best response for player 2. However, $P_{1}$ can only represent a best response for player 1 if player 2 plays action A with probability $\frac{-b}{1-b-c}$. Thus, there is a third set of on-thepath equilibrium strategies such that $(1-\lambda) P_{C C 1}+\lambda P_{F 1}=(1-\lambda) P_{C C 2}+\lambda P_{F 2}=\frac{-b}{1-b-c}$.

We use the above results to analyze which norms are best for fostering cooperation between players. We find that if the probability of forgetting is low enough, cooperation is most-easily supported by cooperative norms (always playing A). On the other hand, if the probability of forgetting is high enough, cooperation can only be sustained under a non-cooperative norm.

Theorem 1: Suppose that $\lambda \leq \frac{-b}{1-b-c}$. Then the strongest incentives for cooperation in the first period of the game are created by a strategy and norm that has equilibrium actions that are fully cooperative.

Proof: Consider a strategy that has fully cooperative equilibrium actions $P_{C C 1}=P_{F 1}=$ $P_{C C 2}=P_{F 2}=1$. In this case, the players can anticipate that if they cooperate in period 1 then their ex ante period 2 payoff will be $\pi^{C}=1$.

Now consider the actions for other states $S=C D, D C$, or $D D$. If $P_{S 1}>\frac{-b}{(1-\lambda)(1-b-c)}-\frac{\lambda}{(1-\lambda)}$ then the best response for player 2 is $P_{S 2}=1$, which in turn would mean that player 1's best response would be $P_{S 1}=1$. Similarly, if $P_{S 1}<\frac{-b}{(1-\lambda)(1-b-c)}-\frac{\lambda}{(1-\lambda)}$ then $P_{S 1}=P_{S 2}=0$ in equilibrium. The third possibility is that both players play a mixed strategy such that $P_{S 1}>\frac{-b}{(1-\lambda)(1-b-c)}-\frac{\lambda}{(1-\lambda)}$.

If $P_{S 1}=P_{S 2}=1$, then the ex ante payoff for the players in state $S$ would still be 1 . Thus, this could not be part of a punishment scheme. If $P_{S 1}=P_{S 2}=0$ the ex ante payoff to each player would be:

$$
\lambda^{2}+\lambda(1-\lambda)(b+c)
$$


where the first term represents the probability that both players are forgetful times the payoff of 1 that they receive in this case, $\lambda(1-\lambda) b$ represents the probability times the payoff that the player receives if they are forgetful but their opponent is not, and $\lambda(1-\lambda) c$ represents the case where their opponent is forgetful and they are not. Note that if neither player is forgetful both players play B and get a payoff of zero.

If, instead, both players play a mixed strategy, the ex ante payoff from that state is $\pi^{M}=\frac{-b c}{1-b-c}$.

To calculate the greatest incentives for cooperation in stage 1, we can take the $e x$ ante payoff the players will receive from cooperating and subtract from it the lowest payoffs that can be imposed on the player in a punishment state. Thus, cooperation in stage 1 can be sustained when $P_{C C 1}=P_{F 1}=P_{C C 2}=P_{F 2}=1$ as long as the incentive to deviate in stage 1 is less than or equal to

$$
1-\min \left[\lambda^{2}+\lambda(1-\lambda)(b+c), \frac{-b c}{1-b-c}\right] .
$$

Suppose instead that $P_{C C 1}=P_{F 1}=P_{C C 2}=P_{F 2}=0$. Following a similar logic as before, three outcomes can be consistent with a modified multiself sequential equilibrium: $P_{S i}=P_{S j}=0, P_{S i}=P_{S j}=1$ or $P_{S i}=P_{S j}=\frac{-b}{(1-\lambda)(1-b-c)}$. The ex ante payoffs from these three strategies is $0,(1-\lambda)^{2}+\lambda(1-\lambda)(b+c)$ and $\frac{-b c}{1-b-c}$ respectively. Thus, the cooperation can be sustained in stage 1 when $P_{C C 1}=P_{F 1}=P_{C C 2}=$ $P_{F 2}=0$ as long as the incentive to deviate in stage 1 is less than or equal to

$$
0-\min \left[(1-\lambda)^{2}+\lambda(1-\lambda)(b+c), \frac{-b c}{1-b-c}\right] .
$$

We can show that the incentives for cooperation in stage 1 are stronger under the fully cooperative norm by comparing (4) and (5). Consider the case where

$$
\begin{gathered}
{\left[(1-\lambda)^{2}+\lambda(1-\lambda)(b+c)\right]<\frac{-b c}{1-b-c} \text {. Then we can see that }(4)>(5) \text { by noting that }} \\
\begin{array}{c}
1-\min \left[\lambda^{2}+\lambda(1-\lambda)(b+c), \frac{-b c}{1-b-c}\right] \geq 1-\lambda^{2}-\lambda(1-\lambda)(b+c) \\
>-1+2 \lambda-\lambda^{2}-\lambda(1-\lambda)(b+c)
\end{array}
\end{gathered}
$$




$$
=-\left[(1-\lambda)^{2}+\lambda(1-\lambda)(b+c)\right]
$$

If $\frac{-b c}{1-b-c}<\left[(1-\lambda)^{2}+\lambda(1-\lambda)(b+c)\right]$, then

$1-\min \left[\lambda^{2}+\lambda(1-\lambda)(b+c), \frac{-b c}{1-b-c}\right] \geq 1-\frac{-b c}{1-b-c}>0-\min \left[(1-\lambda)^{2}+\lambda(1-\lambda)(b+c), \frac{-b c}{1-b-c}\right]$.

We must also compare the best incentives for cooperation when $P_{C C 1}=P_{F 1}=P_{C C 2}$ $=P_{F 2}=1$ compared to the best incentives for cooperation when

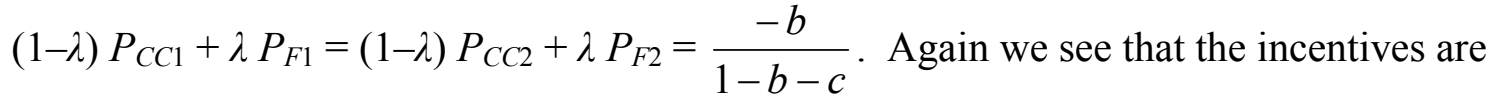
greatest when $P_{C C 1}=P_{F 1}=P_{C C 2}=P_{F 2}=1$, but the details are more tedious. We present the rest of the proof in the Appendix.

\section{Q.E.D.}

While theorem 1 demonstrates that cooperation in period 1 can be best supported by a fully-cooperative norm when the probability of forgetting is not too high, it is not possible to sustain any punishment under this norm when there is too much forgetfulness. However, it is still possible to create a reward and punishment scheme for cooperation in period 1 under other norms even under high levels of forgetfulness. In some cases, having even a small wedge between what the players earn if they cooperate in the first stage compared to what they earn if they deviate can lead to a large gain across the two periods if the stage equilibrium for the game in period 1 is significantly lower than the cooperative payoff in period 1 .

Theorem 2: Suppose that $\lambda>\frac{-b}{1-b-c}$. Then cooperation in period 1 can only be sustained by norms that are not fully cooperative.

Proof: Note that if $\lambda>\frac{-b}{1-b-c}$, it must be that $P_{S i}=1$ in equilibrium if $P_{F(-i)}=1$

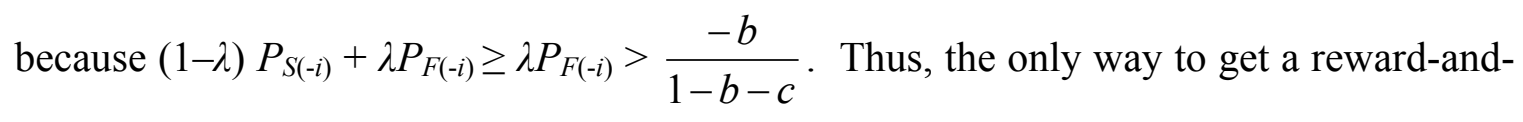
punishment scheme is through a norm that is not fully cooperative. If 
$\frac{-b}{1-b-c}<\lambda \leq \frac{1-c}{1-b-c}$ then one can create a reward and punishment with $P_{F i}=0$ for all players, yielding the incentives in equation (5).

If $\lambda>\frac{\max (-b, 1-c)}{1-b-c}$, it is possible to support a punishment scheme with mixing. We prove this by building a simple, although inefficient, symmetric reward and punishment scheme. Due to the symmetry, we drop subscripts denoting the player's identification. Denote the unconditional probability that each player plays action A as $P=(1-\lambda) P_{S}+\lambda P_{F}$, where $P_{S}$ is the probability with which the players' strategy dictates playing action $A$ given that the player recalls that the state is $S$. The ex ante expected payoffs to each player, entering a given state, is

$$
\pi^{x}=P^{2}+P(1-P)(b+c)
$$

Note that $\frac{\partial \pi^{x}}{\partial P}=b+c+2 P(1-b-c)$. This derivative is $\frac{\partial \pi^{x}}{\partial P}=-b+c$ at $P=\frac{-b}{1-b-c}$. If $-b+c>0$ then a reward and punishment scheme can be created when $P_{C C}=\varepsilon$ for some small $\varepsilon>0, P_{F}=\frac{1}{\lambda}\left(\frac{-b}{1-b-c}-(1-\lambda) \varepsilon\right)$ and $P_{C D}=P_{D C}=P_{D D}=0$. When $-b+c<0$ then a reward and punishment scheme can be created when $P_{C C}=1-\varepsilon$ for some small $\varepsilon>0, P_{F}=\frac{1}{\lambda}\left(\frac{-b}{1-b-c}-(1-\lambda)(1-\varepsilon)\right)$ and $P_{C D}=P_{D C}=P_{D D}=1$. Q.E.D.

We next examine how forgetfulness can change the ability of players to cooperate relative to what is observed under the classical assumption of perfect recall. The following theorem demonstrates that forgetfulness in histories and strategies can either ease or hinder the ability of players to cooperate.

Theorem 3a: Suppose that $-b>c>0$. Then the maximum incentives to cooperate in the first stage of the game are greater for positive levels of $\lambda$ in the neighborhood of zero than they are when $\lambda=0$.

Theorem 3b: Suppose that $-b<c$. Then the maximum incentives to cooperate in the first stage of the game are greater when $\lambda=0$ than they are for positive levels of $\lambda$. 
Proof: Theorem 1 demonstrates that the maximum incentive to cooperate when $\lambda \leq \frac{-b}{1-b-c}$ is given by $1-\min \left[\lambda^{2}+\lambda(1-\lambda)(b+c), \frac{-b c}{1-b-c}\right]$. Also, $\lambda^{2}+\lambda(1-\lambda)(b+c)<\frac{-b c}{1-b-c}$ when $c>0 .{ }^{11} \quad$ If we take the derivative $\frac{\partial\left[1-\lambda^{2}-\lambda(1-\lambda)(b+c)\right]}{\partial \lambda}=-2 \lambda(1-b-c)-(b+c)$. This is negative if $-b<c$, and it is positive in the neighborhood of $\lambda=0$ if $-b>c$. The last thing to consider is what happens when $\lambda>\frac{-b}{1-b-c}$. In this case, the maximum feasible wedge between the reward and punishment payoffs is $1-\frac{-b c}{1-b-c}$. However, this is less than 1 , and does not change with the level of forgetfulness.

Q.E.D.

Note that forgetfulness can make cooperation easier despite the fact that a player that was cheated upon might forget to punish the player that cheated; this is due to two countervailing effects. First, the player who cheated might forget that they were being punished and play A even when the optimal action, given that they cheated, would be to play B. This effect was illustrated with concrete numbers in the example of Section 3.1. Second, the player that was cheated upon will also play responses that are not optimal given that the other player cheated. While this hurts the one meting out the punishment, it also hurts the player who cheated.

While this second effect cannot be demonstrated using the second-stage game represented in Figure 1, a game whose second-stage is presented in Figure 3 offers a concrete example. The minimax payoff for this game is $-5 / 2$. Delivering the minimax requires mixing between actions $\mathrm{A}$ and $\mathrm{C}$ with probability $1 / 2$ each. The best response to this action is to play either B or C. However, any player delivering the minimax would get an expected payoff between -50 and -47.5 , depending on which action the deviating

\footnotetext{
${ }^{11} c>0$ in either case, since $-b$ is always positive. Thus, $-b c /(1-b-c)>0$. To see that $\lambda^{2}+\lambda(1-\lambda)(b+c)<$ $-b c /(1-b-c)$, note that $\lambda^{2}+\lambda(1-\lambda)(b+c)$ is quasi-concave. Thus, the maximum of $\lambda^{2}+\lambda(1-\lambda)(b+c)$ in the interval $\lambda \in[0,-b /(1-b-c)]$ must occur at one of the end points. At $\lambda=0, \lambda^{2}+\lambda(1-\lambda)(b+c)=0$. At $\lambda=$ $-b /(1-b-c), \lambda^{2}+\lambda(1-\lambda)(b+c)=-b c /(1-b-c)$. If $c<0, \lambda^{2}+\lambda(1-\lambda)(b+c)>-b c /(1-b-c)$ in this interval, which means that forgetfulness neither helps nor hurts the ability for the players to cooperate.
} 
player chooses. However, the player meting out the punishment could instead earn higher profits if they always played $\mathrm{C}$. Thus, the minimax is not credible.

The minimax can be credible, however, if the players are forgetful. Consider this strategy and norm combination (played by both players) for a second stage of the game.

(a) If the player forgets, play A.

(b) If the player recalls that both players cooperated in stage 1, play A.

(c) If the player recalls that there was a deviation in the first stage, play $\mathrm{C}$.

Note that if the player recalls cooperation then (A, A) forms a Nash equilibrium. Thus, if the players recall cooperation then there is no incentive to deviate. Similarly, if the player is forgetful, A is still a best response since they can expect that their opponent will play A in equilibrium. We need to also confirm that a player who recalls a deviation will follow this strategy. If they follow their strategy, they will earn a payoff of $-5 \lambda$. If they deviate to action A their payoff will instead be $\lambda-100(1-\lambda)=-100+101 \lambda<-5 \lambda$ as long as $\lambda<100 / 106$, while if they deviate to action B their payoff will be $0 \lambda-5(1-\lambda)=$ $-5+5 \lambda<-5 \lambda$ as long as $\lambda \leq 1 / 2$.

Imagine now that $\lambda=1 / 2$. Then the payoff to the deviating player in the second stage is indeed the minimax payoff of $-5 / 2$. While generally the level of forgetfulness will not be set at the perfect level to deliver the most severe punishment, this example nevertheless demonstrates that forgetfulness can make credible punishments that are more-severe than the most-severe punishments one could obtain under perfect recall. Note that while forgetfulness allows the player to credibly deliver a harsher punishment to an opponent who deviates, in equilibrium this more-severe threat means that this costly punishment is never endured. Thus, the ability to commit to being ignorant of a deviation - leading to pain on both sides - allows the players to obtain higher profits than they would obtain under perfect recall. ${ }^{12}$

\footnotetext{
${ }^{12}$ There is some precedence for ignorance about an opponent's actions leading to higher profits: Coughlan and Mantrala (1994) use a dynamic pricing game to show that prices are higher if firms believe that there will be a stronger price retaliation than actually exists. In their model, the players are able to fit observed behavior with the mistaken impressions of the behavior of their competitors. However, both firms can be better off with both firms having uncertainty over what the strategies of their opponents are rather than having their strategies laid bare.
} 
While the punishments that arise when players forget strategies and histories can be severe enough to make cooperation easier to sustain, it can also entrench inefficiencies. In fact, the risk to a player of inflicting great harm to themselves when they forget that they are being punished may be severe enough that equilibrium payoffs can be below the minimax.

We illustrate this possibility with the two-stage game pictured in Figure 4. Note that the minimax payoff of this game is zero. However, when $\lambda \in[1 / 3,1 / 2]$, there exists a sequential equilibrium with payoffs below zero where both players play the following strategy and norm: each player plays A in the first period. In the second period, both players play $\mathrm{X}$ if the player remembers both players playing $\mathrm{A}$ in the previous period or if the player has forgotten what happened previously; otherwise they play Y. To confirm that this strategy is an equilibrium, note by inspection that on-the-path it does not pay for a player to deviate from playing $X$ in the second period because their stage payoff will be 1 , which is the maximum possible stage payoff. Further, if the player recalls a deviation in the first period then the player should play $\mathrm{Y}$ as long as $\lambda \leq 5 / 6$. This is because their payoff of playing $\mathrm{Y}$ is 0 , while their payoff of playing $\mathrm{X}$ is $\lambda-5(1-\lambda)$. We can then examine what would happen in the first period given these second period strategies. The expected total payoff if the player plays $\mathrm{A}$ is $-2+1=-1$. If the player deviates, on the other hand, their expected payoff is $0+\lambda^{2}-5 \lambda(1-\lambda)$. Thus, the players will not deviate in period 1 as long as $-1 \geq-5 \lambda+6 \lambda^{2} \rightarrow 0 \geq(3 \lambda-1)(2 \lambda-1)$, which corresponds to the interval $\lambda \in[1 / 3,1 / 2]$. Thus, the players will not deviate in the first period, and the equilibrium payoff is -1 even though the minimax is equal to 0 .

Finally, we note that once there is enough forgetfulness, cooperation becomes more difficult to sustain as the players become more forgetful. This occurs because once there is enough forgetfulness, the link between the two periods becomes so weak that there is no effective way to punish deviators in the first period. This can be formalized by the following theorem:

Theorem 4: The difference between the maximum and minimum second-period payoffs that can be implemented in an equilibrium goes to zero as $\lambda \rightarrow 1$. 
Proof: Denote the total (unconditional) probability of playing action $\mathrm{A}$ in the state where the players cooperated in period 1 as $P_{R 1}$ and $P_{R 2}{ }^{13}$ Denote the total (unconditional) probability of playing action A in the state where a deviation is recalled as $P_{P 1}$ and $P_{P 2}$. The ex ante payoff to player $i$ in any state $S$ is

$$
\pi^{S}=P_{S i} P_{S(-i)}+P_{S i}\left(1-P_{S(-i)}\right) b+P_{S(-i)}\left(1-P_{S i}\right) c
$$

Note that $\left|P_{R i}-P_{P i}\right| \leq(1-\lambda)$. Thus, $P_{R i} \stackrel{\lambda \rightarrow 1}{\longrightarrow} P_{P i}$. It immediately follows from equation (7) that $\pi^{i}{ }_{R} \stackrel{\lambda \rightarrow 1}{\longrightarrow} \pi^{i}{ }_{P}$ for both players. Thus the difference in the maximum and minimum payoffs that can be supported in the second period go to zero, and the ability to support cooperation in the first stage of the game disappears, as the level of forgetfulness gets close to 1 .

Q.E.D.

\section{Forgetting Histories, but Not Strategies}

We make only one distinction between modeling games where players forget both histories and strategies and games where players forget histories but not strategies: when players forget their past actions, but not their strategies, their current decisions are based upon the strategies they coordinated across all incarnations during pre-play planning period. Confirming that a set of strategies form an equilibrium requires (1) confirming that the strategies of each player, across their various incarnations, form a Nash equilibrium, and (2) confirming that the strategies form a modified multiselves sequential equilibrium, as defined earlier in the paper.

Note that all equilibria of games where players forget histories but not strategies are also equilibria that could occur if players forget both histories and strategies. ${ }^{14}$ However, the converse is not true: there are some equilibria that arise when players forget histories and strategies that are not equilibria if players forget histories but not strategies.

The contrast between analyzing games where players forget histories and strategies and games where players forget histories but recall their strategy is that in the

\footnotetext{
${ }^{13} P_{R i} \equiv(1-\lambda) P_{C C i}+\lambda P_{F i}$

${ }^{14}$ Set the norm as the actions players would choose to specify in the planning stage for the contingencies where they forgot the past.
} 
latter case the strategy the players follow after they forget is chosen by each player before they start the game. One important consequence is that it becomes possible for the players to hard-code messages to their future selves about what actions they should take when they are forgetful. Thus, for example, a player who wants to cheat on an agreement in an early stage of the game can hard-code into their strategy to play an optimal deviation plan, which includes an optimal defense of their strategies. For example, it is no longer possible to impose punishments that deliver below-minimax payoffs when players remember their strategies because a player who deviates from an agreed upon strategy can program their own future actions (via their strategies) in a way that allows them to receive at least the minimax. By contrast, the actions that players take after they forget in games where the players forget their strategies are set by convention (and, thus, never in the players control).

However, forgetfulness in histories but not strategies can still aid cooperation by making credible severe punishments that are very expensive to both players. We illustrate this by returning to the game whose second-stage is presented in Figure 3. We analyzed properties of this game in Section 3, where we noted that meting out the minimax payoff of $-5 / 2$ is not credible under perfect recall, but is credible when players forget their strategies and histories.

It turns out that this punishment is also credible when players forget their histories but not their strategy. In essence, players will follow the same strategies as in Section 3; however, the norm of actions chosen by players if they forget the history of the game is now chosen by players at the start of the game. Thus, we need to confirm that there are no profitable deviations from this strategy.

Consider this strategy (played by both players) game:

(a) Cooperate in the first stage of the game.

(b) Play A if (1) the player recalls that both players cooperated in stage 1, or (2) if the player cannot recall what happened in the first stage.

(c) Play $\mathrm{C}$ if the player recalls that there was a deviation in the first stage.

In Section 3.2 we proved that these strategies form a modified multiselves sequential equilibrium. To confirm that this is an equilibrium when players always recall their strategies, we must also show that the player will not choose a different strategy for the 
overall game. We see from the previous analysis that each of the contingent actions in the second period is optimal given the prescribed equilibrium, and given the second stage strategies, it is not optimal to deviate in the first stage. However, a player who deviated in the first period is also able to hard-code a particular action into their deviation strategy. Thus, a player who deviates can deviate in a way that causes them to act as if they have recall of their own past actions. ${ }^{15}$ In Section 3.2, we saw that a player who is planning on deviating and not cooperating in the first stage should play action $\mathrm{C}$ in the second stage as long as $\lambda \leq 1 / 2$. Thus, if the player deviates from the strategy, it will be in a way that deviates in the first period and then plays $\mathrm{C}$ in the second period regardless of whether they remember or forget. Imagine now that $\lambda=1 / 2$. Then the payoff to the deviating player in the second stage is the minimax payoff of $-5 / 2$. As before, we note that the level of forgetfulness will generally not equal the level that delivers the most severe punishment. This is not our goal here. Rather, the example is merely meant to illustrate that forgetfulness of histories but not strategies can make cooperation easier by making severe punishments credible.

\section{Conclusion}

This paper expands the game theory literature by removing the assumption of perfect recall in multi-player strategic games. Games with imperfect recall can be analyzed using a modified classical game-theoretic framework. We distinguish between forgetting histories and forgetting strategies, and explain how classical game theoretic models and equilibrium concepts should be adapted to accommodate imperfect recall. We find that imperfect recall can sometimes ease cooperation, although it can also reinforce inefficient outcomes or make cooperation more difficult in some games. In games with forgetfulness in histories and strategies, forgetfulness has countervailing effects on cooperation: the diminished ability to punish reduces the ability to cooperate, while the diminished ability to effectively deviate and the increased credibility of severe punishments aids cooperation. Either effect may dominate. In fact, the latter effects may be large enough that there can exist equilibria with on-the-path payoffs that are below the

\footnotetext{
${ }^{15}$ The action that their strategy prescribes after they are forgetful cannot account for their rival's actions, however. While this does not affect the current example, there are other situations where this can matter.
} 
minimax. Our examination of games where players forget histories but not strategies highlights that even if players always remember their strategies, forgetfulness can still increase the ease of cooperation if forgetfulness allows players to punish deviations with more severe punishments than they would be willing to mete out under perfect recall.

There are several business applications of the results discussed in the current research. For example, firms make large investments in information technology to document prior strategies and actions. These investments are made with the expectation that a better recall will lead to better decisions which in turn will result in higher profits for the firm. However, a key result of this paper is that forgetfulness can help in cooperation. This would then imply that it is not always in the firm's best interest to document history. It would be insightful to study what the extent of this investment in information technology should be. Also, in a competitive setting would all firms invest the same in documenting past actions and strategies? 


\section{Appendix}

\section{Completion of Proof of Theorem 1}

We must also compare that the incentives are greatest when $P_{C C 1}=P_{F 1}=P_{C C 2}$ $=P_{F 2}=1$, but the details are more tedious. Thus, we present the rest of the proof in this Appendix.

We compare the best incentives for cooperation when $P_{C C 1}=P_{F 1}=P_{C C 2}=P_{F 2}=1$ compared to the best incentives for cooperation when

$(1-\lambda) P_{C C 1}+\lambda P_{F 1}=(1-\lambda) P_{C C 2}+\lambda P_{F 2}=\frac{-b}{1-b-c}$. We allow the two players to have asymmetric strategies. While this can create unequal punishments for the two players, it is possible that in the unmodeled first stage one player needs greater incentives for cooperation than the other player. That said, we find that the case of fully cooperative norms still yields the best incentives for cooperation.

In the case where the players mix after cooperation, it must be that the players do not mix after there is a deviation in any scheme which punishes such a deviation. For logic similar to that in the text, it must be that the players' strategies must be such that either both players play A with probability 1 or both play A with probability 0 if the players recall a deviation.

Case 1: Both players play A if they recall that they are in a punishment state

In this case, the players will play action $\mathrm{A}$ with the ex ante probability $\left(1-\lambda+\lambda P_{F i}\right)$ and they will play action $\mathrm{B}$ with the ex ante probability $\lambda\left(1-P_{F i}\right)$. Note that in order for

$$
\begin{gathered}
(1-\lambda) P_{C C 1}+\lambda P_{F 1}=(1-\lambda) P_{C C 2}+\lambda P_{F 2}=\frac{-b}{1-b-c} \text { it must also be the case that } \\
\lambda\left(1-P_{F i}\right) \leq \frac{1-c}{1-b-c} \\
1-\lambda+\lambda P_{F i} \geq \frac{-b}{1-b-c} .
\end{gathered}
$$

The ex ante punishment profit for player $i$ (playing against player $j$ ) is then

$$
\left(1-\lambda+\lambda P_{F i}\right)\left(1-\lambda+\lambda P_{F j}\right)+\left(1-\lambda+\lambda P_{F i}\right) \lambda\left(1-P_{F j}\right) \mathrm{b}+\lambda\left(1-P_{F i}\right)\left(1-\lambda+\lambda P_{F j}\right) \mathrm{c}
$$

We can then compare the maximum incentives to cooperate with those that we can have under the fully cooperative norm: 


$$
\begin{aligned}
& 1-\lambda^{2}-\lambda(1-\lambda)(b+c) \stackrel{?}{>} \\
& \quad \frac{-b c}{1-b-c}-\left(1-\lambda-\lambda P_{F i}\right)\left(1-\lambda-\lambda P_{F j}\right)-\left(1-\lambda-\lambda P_{F i}\right) \lambda\left(1-P_{F j}\right) b-\lambda\left(1-P_{F i}\right)\left(1-\lambda-\lambda P_{F j}\right) c
\end{aligned}
$$

Which can be rearranged as

$$
2+\frac{b c}{1-b-c}+\lambda^{2}\left[\left(1-P_{F i}\right)\left(1-P_{F j}\right)-1\right](1-b-c)-\lambda(b+c)-\lambda\left(1-P_{F j}\right)(1-b)-\lambda\left(1-P_{F i}\right)(1-c) \stackrel{?}{0}
$$

The second derivative of the left-hand side with respect to $\lambda$ is $2(1-\mathrm{b}-\mathrm{c})\left(-P_{F i}-P_{F j}+P_{F i} P_{F j}\right)$, which is negative. Thus, this function is quasi-convex, meaning that the minimum must occur at one of the endpoints in the region we consider.

At $\lambda=0$, the left side of (a.4) is $2-\frac{-b c}{1-b-c}>0$.

At $\lambda=\frac{-b}{1-b-c}$, the left side of (a.4) is

$$
\frac{2-2 b-2 c+2 b c+b^{2}\left(1-P_{F i}\right)\left(1-P_{F j}\right)}{1-b-c}-\lambda\left(1-P_{F j}\right)(1-b)-\lambda\left(1-P_{F i}\right)(1-c)
$$

If $c \geq \mathrm{b}$, we can use the inequality $\lambda\left(1-P_{F i}\right) \leq \frac{1-c}{1-b-c}$ and note that

$$
\begin{aligned}
(\mathrm{a} .5) & \geq \frac{2-2 b-2 c+2 b c+b^{2}\left(1-P_{F i}\right)\left(1-P_{F j}\right)-(1-c)(1-b)-(1-c)(1-c)}{1-b-c} \\
& =\frac{b^{2}\left(1-P_{F i}\right)\left(1-P_{F j}\right)+(c-b)(1-c)}{1-b-c} .
\end{aligned}
$$

Each term is positive on inspection. If $\mathrm{c}<\mathrm{b}$, then we plug $\lambda=\frac{-b}{1-b-c}$ into (a.5)

$$
\begin{aligned}
(\mathrm{a} .5) & =2+\frac{2 b c+b^{2}\left(1-P_{F i}\right)\left(1-P_{F j}\right)+b(1-b)\left(1-P_{F j}\right)+b(1-c)\left(1-P_{F i}\right)}{1-b-c} \\
& =2+\frac{b c+b^{2}\left(1-P_{F i}\right)\left(1-P_{F j}\right)+2 b-b^{2}-b(1-b) P_{F j}-b(1-c) P_{F i}}{1-b-c} \\
& =2+\frac{b c-P_{F i} b^{2}+P_{F i} P_{F j} b^{2}+2 b-b P_{F j}-b P_{F i}+b c P_{F i}}{1-b-c} \\
& >\frac{2-2 c+\left(b^{2}-P_{F i} b^{2}\right)+P_{F i} P_{F j} b^{2}+b c P_{F i}}{1-b-c}
\end{aligned}
$$




$$
\geq \frac{2(1-c)+P_{F i} P_{F j} b^{2}+b c P_{F i}}{1-b-c}
$$

Each term is positive.

Case 2: Both players play B if they recall that they are in a punishment state

In this case, the players will play action A with the ex ante probability $\lambda P_{F i}$ and they will play action $\mathrm{B}$ with the ex ante probability $1-\lambda P_{F i}$. The ex ante punishment profit for player $i$ (playing against player $j$ ) is then

$$
\lambda^{2} P_{F i} P_{F j}+\lambda P_{F i}\left(1-\lambda P_{F j}\right) \mathrm{b}+\left(1-\lambda P_{F i}\right) \lambda P_{F j} \mathrm{c}
$$

We can find the values of $P_{F i}$ and $P_{F j}$ that minimize this payoff:

$$
\frac{\partial(a .6)}{\partial P_{F i}}=\lambda\left[\lambda P_{F 2}(1-b-c)+b\right]<0 .
$$

The last inequality occurs because $\lambda P_{F i} \leq \frac{-b}{1-b-c}$ (or else mixing would not be possible on-the-equilibrium path). This implies that the punishment is minimized when $P_{F i}=1$.

$$
\frac{\partial(a .6)}{\partial P_{F j}}=\lambda\left[\lambda P_{F 1}(1-b-c)+c\right]=\lambda[\lambda(1-b-c)+c] .
$$

This is non-positive if $\lambda \leq \frac{-c}{1-b-c}$, in which case we should set $P_{F j}=1$. If $\lambda>\frac{-c}{1-b-c}$ this is positive, so we want to set $P_{F j}$ as low as possible. This is $P_{F j}=0$ if $\lambda \leq \frac{1-c}{1-b-c}$, but $\lambda P_{F j}=\frac{-b}{1-b-c}-1+\lambda$ if $\lambda>\frac{1-c}{1-b-c}$. (This reflects the minimum value for $P_{F j}$ that allows mixing on the equilibrium path.

Case $2 \mathrm{~A}: \lambda \leq \frac{-c}{1-b-c}$

Plugging in $P_{F i}=P_{F j}=1$, the punishment in (a.6) becomes $\lambda^{2}+\lambda(1-\lambda) \mathrm{b}+\lambda(1-\lambda) \mathrm{c}$, which is the same as the punishment payoff we get under the fully cooperative norm. This makes sense, since we have the same cooperative norm in this case. However, the "reward" payoff is greater when $P_{C C 1}=P_{C C 2}=1$ compared to when $(1-\lambda) P_{C C 1}+\lambda P_{F 1}=$ $(1-\lambda) P_{C C 2}+\lambda P_{F 2}=\frac{-b}{1-b-c}$. 
Case 2B: $\lambda \in\left[\frac{-c}{1-b-c}, \min \left(\frac{1-c}{1-b-c}, \frac{-b}{1-b-c}\right)\right]$.

Plugging in $P_{F i}=1$ and $P_{F j}=0$ to (a.6) yields a punishment of $\lambda b$. We can then compare the maximum incentives to cooperate with those that we can have under the fully cooperative norm:

$$
\begin{gathered}
1-\lambda^{2}-\lambda(1-\lambda)(b+c) \stackrel{?}{\frac{?}{1-b-c}-}-\lambda b \\
\Rightarrow 1-\lambda^{2}(1-b-c)-\lambda c-\frac{-b c}{1-b-c} \stackrel{?}{>} 0
\end{gathered}
$$

If we take the second derivative of (a.7) with respect to $\lambda$ confirms that this is a quasiconcave. Thus, the minimum must occur at one of the edges of the interval we are considering. Note that if the true lower bound is 0 or upper bound is $-b /(1-b-c)$ then we are looking at a wider span of values for $\lambda$, which can only have a lower minimum.

$$
\begin{aligned}
& \text { At } \lambda=\frac{-c}{1-b-c},\left(\text { a.7) is } 1-\frac{-b c}{1-b-c}>0 .\right. \\
& \text { At } \lambda=\frac{1-c}{1-b-c},\left(\text { a.7) is } 1+\frac{b c-1+c}{1-b-c} \geq 1-\frac{1-b-c}{1-b-c}=0 .\right.
\end{aligned}
$$

Case $2 \mathrm{C}: \lambda \in\left[\frac{1-c}{1-b-c}, \frac{-b}{1-b-c}\right]$.

Plugging in $P_{F i}=1$ into equation (a.6) and then comparing with the incentives for cooperation in stage 1 when $P_{C C 1}=P_{F 1}=P_{C C 2}=P_{F 2}=1$ yields

$$
1-\lambda^{2}(1-b-c)-\lambda(b+c)-\frac{-b c}{1-b-c}+\lambda^{2} P_{F j}(1-b-c)+\lambda b+\lambda P_{F j} c>?
$$

We can take the second derivative of the left side (a.9) with respect to $\lambda$ and we can confirm that it is negative, implying that this is a quasi-concave function. Thus, we only need to check this condition at the two end points.

At $\lambda=\frac{1-c}{1-b-c}, \lambda P_{F j}=\frac{-b}{1-b-c}-1+\lambda=0$. This gives the same calculations as we performed in (a.8).

At $\lambda=\frac{-b}{1-b-c}, \lambda P_{F j}=\frac{-2 b}{1-b-c}-1$

Plugging these into equation (a.9) yields 


$$
1+\frac{-b^{2}+b^{2}+b c+b c+2 b^{2}+b(1-b-c)-b^{2}-2 b c}{1-b-c}-c=(1-c)+\frac{b(1-c)}{1-b-c}>0
$$

Q.E.D. 
Figure 1:

\begin{tabular}{|c|c|c|}
\hline & $\mathrm{A}$ & $\mathrm{B}$ \\
\hline $\mathrm{A}$ & 1,1 & $\mathrm{~b}, \mathrm{c}$ \\
\hline $\mathrm{B}$ & $\mathrm{c}, \mathrm{b}$ & 0,0 \\
\hline
\end{tabular}

Figure 2: Two Stage Game

Stage One
\begin{tabular}{|c|c|c|}
\hline & Coop & Fink \\
\hline Coop & 4,4 & $-1,5.5$ \\
\hline Fink & $5.5,-1$ & 0,0 \\
\hline
\end{tabular}

\begin{tabular}{|c|c|c|}
\multicolumn{1}{c}{ Stage Two } \\
\begin{tabular}{|c|c|c|}
\hline & A & B \\
\hline A & 1,1 & $-4,1 / 2$ \\
\hline B & $1 / 2,-4$ & 0,0 \\
\hline
\end{tabular}
\end{tabular}

Figure 3:

\begin{tabular}{|c|c|c|c|}
\hline & $\mathrm{A}$ & $\mathrm{B}$ & $\mathrm{C}$ \\
\hline $\mathrm{A}$ & 1,1 & $-100,0$ & $-100,-5$ \\
\hline $\mathrm{B}$ & $0,-100$ & $0.5,0.5$ & $-5,5$ \\
\hline $\mathrm{C}$ & $-5,-100$ & $5,-5$ & 0,0 \\
\hline
\end{tabular}

The minimax payoff is $-5 / 2$. A player wishing to impose the minimax on their opponent must mix, playing $A$ with probability $1 / 2$ and $C$ with probability $1 / 2$. This gives the other player a payoff of -5 if they play action $\mathrm{A}$ or $-5 / 2$ if they play $\mathrm{B}$ or $\mathrm{C}$. Playing B with a higher probability would increase the payoffs to a player playing either $\mathrm{B}$ or $\mathrm{C}$, while playing $\mathrm{A}$ or $\mathrm{C}$ with a higher probability increases the payoff to an opponent playing $\mathrm{B}$ or $\mathrm{C}$, respectively, (or an opponent playing A) so this is the minimax. Thus, playing either $\mathrm{B}$ or $\mathrm{C}$ is a best response to this minimax.

Figure 4:

Stage One
\begin{tabular}{|c|c|c|}
\hline & A & B \\
\hline A & $-2,-2$ & $-5,0$ \\
\hline B & $0,-5$ & 0,0 \\
\hline
\end{tabular}

Stage Two
\begin{tabular}{|c|c|c|}
\hline & $\mathrm{X}$ & $\mathrm{Y}$ \\
\hline $\mathrm{X}$ & 1,1 & $-5,0$ \\
\hline $\mathrm{Y}$ & $0,-5$ & 0,0 \\
\hline
\end{tabular}




\section{Bibliography:}

Alavi, Maryam and Dorothy E. Leidner, "Review: Knowledge Management and Knowledge Management Systems: Conceptual Foundations and Research Issues," MIS Quarterly, 25(1), 2001, pp. 107-136.

Alpern, Steve, "Games with Repeated Decisions," SIAM Journal of Control and Optimization, 1988, 26(2), 468-477.

Anderlini, Luca, Dino Gerardi and Roger Lagunoff, “A "Super” Folk Theorem for Dynastic Repeated Games," Economic Theory, forthcoming.

Argote, Linda, Bill McEvily and Ray Reagans, "Managing Knowledge in Organizations: An Integrative Framework and Review of Emerging Themes," Management Science, 49(4), 2003, pp. 571-582.

Aumann, Robert, Sergiu Hart and Motty Perry, "The Absent-Minded Driver," Games and Economic Behavior, 20(1), 1997, pp. 102-116.

Battigalli, Pierpaolo, "Dynamic Consistency and Imperfect Recall," Games and Economic Behavior, 20(1), 1997, pp. 31-50.

Benkard, Lanier, "Learning and Forgetting: The Dynamics of Aircraft Production," The American Economic Review 90(4), 2000, pp.1034-1054.

Bernheim, B. Douglas and Raphael Thomadsen, "Memory and Anticipation," Economic Journal, 105(503), 2005, pp. 271-304.

Besanko, David, Ulrich Doraszelski, Yaroslav Kryukov and Mark Satterthwaite, "Learning-by-Doing, Organizational Forgetting, and Industry Dynamics," mimeo, February 2007.

Binmore, Ken, 1992, Fun and Games: A Text on Game Theory, D.C. Heath and Company, Lexington, MA.

Chen, Yuxin, Ganesh Iyer and Amit Pazgal, "Limited Memory and Market Competition," mimeo, July 2005.

Connor, J.M., 2001, Global Price Fixing: Our Customers are the Enemy. Klewer Acadmic Publishers, Boston.

Coughlan, Anne T. and Murali K. Mantrala (1994): "Dynamic Competitive Retail Pricing Behavior with Uncertainty and Learning," Managerial and Decision Economics, 15, 320 . 
Gilboa, Itzhak, "A Comment on the Absent Minded Driver Paradox," Games and Economic Behavior, 20, 1997, 25-30.

Grove, Adam and Joseph Halpern, "On the Expected Value of Games with Absentmindedness," Games and Economic Behavior, 20, 1997, 51-65.

Halpern, Joseph, "On Ambiguities in the Inerpretation of Game Trees," Games and Economic Behavior, 20(1), 1997, pp. 66-96.

Lipman, Bart, "More Absentmindedness," Games and Economic Behavior, 20(1), 1997, 97-101.

Mas-Colell, Andreu, Michael D. Whinston and Jerry R. Green, 1995, Microeconomic Theory, Oxford University Press, New York.

Mehta, Nitin, Surendra Rajiv and Kannan Srinivasan, "Role of Forgetting in MemoryBased Choice Decisions: A Structural Model," Quantitative Marketing and Economics, 2(2), 2004, pp. 107-140.

Mullainathan, Sendhil, "A Memory-Based Model of Bounded Rationality," The Quarterly Journal of Economics, 117(3), 2002, pp.735-774.

Neumann, John von and Oskar Morgenstern, Theory of Games and Economic Behavior, John Wiley and Sons, New York. 1953.

Ofek, Elie, Muhamet Yildiz and Ernan Haruvy, "The Impact of Prior Decisions on Subsequent Valuations in a Costly Contemplation Model." Management Science, 2007, 53(8), 1217-1233.

Piccione, Michele, and Ariel Rubinstein, "On the Interpretation of Decision Problems with Imperfect Recall,” Games and Economic Behavior, 20(1), 1997, pp. 3-24.

Rubinstein, Ariel, "Finite Automata Play the Repeated Prisoner's Dilemma," Journal of Economic Theory, 1986, 39, 83-96.

Segal, Uzi, "Don't Fool Yourself to Believe You Won't Fool Yourself Again," Economics Letters, 67, 2000, 1-3.

Stein, E. W., "Organizational Memory: Review of Concepts and Recommendations for Management," International Journal of Information Management, 15(2), 1995, pp. 1732. 